\title{
Research Article Dynamics of a Discretization Physiological Control System
} Xiaohua Ding and Huan Su

Received 31 May 2006; Revised 19 November 2006; Accepted 20 November 2006

We study the dynamics of solutions of discrete physiological control system obtained by Midpoint rule. It is shown that a sequence of Hopf bifurcations occurs at the positive equilibrium as the delay increases and we analyze the stability of the solution of the discrete system and calculate the direction of the Hopf bifurcations. The numerical results are presented.

Copyright (c) 2007 X. Ding and H. Su. This is an open access article distributed under the Creative Commons Attribution License, which permits unrestricted use, distribution, and reproduction in any medium, provided the original work is properly cited.

\section{Introduction}

Recently, there has been an interest in the so-called dynamical diseases, which correspond to physiological disorders for which a generally stable control system becomes unstable [1-5]. Here, the study of the dynamical features of the corresponding model such as equilibria, their local stability characteristics and bifurcation behaviors is extremely useful related to the dynamics of mathematical models of various biological systems and other applications. In this paper, we research an arterial $\mathrm{CO}_{2}$ control system [1], which may be described by

$$
\dot{p}(t)=\gamma-\frac{\beta v_{m} p(t) p^{n}(t-\tau)}{\theta^{n}+p^{n}(t-\tau)}, \quad t \geq 0
$$

where $p$ is the arterial $\mathrm{CO}_{2}$ concentration, $\gamma$ is the $\mathrm{CO}_{2}$ production rate, $\tau$ is the time between oxygenation of blood in the lungs and stimulation of chemoreceptors in the brainstem, $v_{m}$ is the maximum ventilation, $\theta$ and $n$ are parameters adjusted to fit experimental observations, and $\beta$ is a constant. The equation reproduces certain qualitative features of normal and abnormal respiration. 
Considering the need of scientific computation and real-time simulation, our interest is focused on the behaviors of discrete dynamics system corresponding to (1.1). Most of this time, it is desirable that a difference equation, which is derived from a differential equation, preserves the dynamical features of the corresponding continuous-time model. That is, the discrete-time model is "dynamically consistent" with the continuous-time model.

In [6], Wulf and Ford show that, if applying Euler forward method to solve the delay differential equation, then the discrete scheme is "dynamically consistent" with the continuous-time model. It means that for all sufficiently small step sizes, the discrete model undergoes a Hopf bifurcation of the same type with the corresponding continuous-time model, and the bifurcation point $\lambda_{h}$ of the discrete model is $O(h)$ close to the bifurcation point $\lambda_{*}$, which corresponds to the continuous-time model.

In this paper, we choose Midpoint rule [7-9] to make the discretization for system (1.1), this method can be considered as perturbation of the Euler forward method. With a similar analysis in [6], it is known that the discrete model is "dynamically consistent" with the continuous-time model for all sufficiently small step sizes, and we can expect the bifurcation point $\lambda_{h}$ of the discrete model is $O\left(h^{2}\right)$ close to the bifurcation point $\lambda_{*}$ of the corresponding continuous-time model due to the Midpoint rule have the convergence of 2 -order [ 7,9$]$. On the other hand, the Midpoint rule is a symplectic method $[8,9]$, it may preserve some important properties of the solutions of the original dynamical system.

The paper is organized as follows. In Section 2, we use the Hopf bifurcation theory of discrete system [10-12] to investigate the stability of equilibrium and the existence of the local Hopf bifurcations at the equilibrium. In Section 3, direction and stability of the Hopf bifurcation are established. In Section 4, some numerical simulations are provided to illustrate the results found. At last, roughly, we apply our results to explain the symptom of respiration.

\section{Stability of the positive equilibrium and local Hopf bifurcations}

In this section, we will see that, when Midpoint rule is applied to system (1.1), it gives rise to a discrete dynamics system (2.8) and we study the stability of the positive equilibrium and the existence of local Hopf bifurcations of system (2.8), which inherits certain dynamics of system (1.1) [13].

Under transformation $p(t)=\theta x(t),(1.1)$ becomes

$$
\dot{x}(t)=a-b \frac{x(t) x^{n}(t-\tau)}{1+x^{n}(t-\tau)},
$$

where $a=\gamma / \theta, b=\beta v_{m}$. Let $u(t)=x(\tau t)$. Then there is $\dot{x}(t)=\dot{u}(t / \tau)(1 / \tau)$. Inserting them into (2.1), we have

$$
\frac{1}{\tau} \dot{u}\left(\frac{t}{\tau}\right)=a-b \frac{u(t / \tau) u^{n}(t / \tau-1)}{1+u^{n}(t / \tau-1)}
$$


So (2.1) can be rewritten as

$$
\dot{u}(t)=a \tau-b \tau \frac{u(t) u^{n}(t-1)}{1+u^{n}(t-1)} .
$$

Thanks to the role of system (1.1) in practice, we only take an interest in the positive equilibrium point of (1.1). Without loss of generality, assume that $\tilde{u}$ is the positive equilibrium point of (2.3), that is,

$$
a \tau-b \tau \frac{\tilde{u}^{n+1}}{1+\tilde{u}^{n}}=0
$$

which has a unique positive equilibrium point.

In fact, by defining function $F(x):=b x^{n+1}-a x^{n}-a$, from

$$
F^{\prime}(x)=(n+1) b x^{n}-a n x^{n-1}= \begin{cases}<0, & 0<x<\frac{a n}{b(n+1)} \\ >0, & x>\frac{a n}{b(n+1)}\end{cases}
$$

and $F(0)=-a<0$, it follows that (2.3) has a unique positive equilibrium point.

If we apply the Midpoint rule to autonomous delay differential equations

$$
\dot{u}=f(u(t), u(t-1)), \quad t \geq 0 ; u(t)=\phi(t),-1 \leq t \leq 0,
$$

we could get

$$
\begin{gathered}
u_{k+1}=u_{k}+h f\left(\frac{u_{k}+u_{k+1}}{2}, \frac{u_{k-m}+u_{k-m+1}}{2}\right), \quad k \geq 0, \\
u_{k}=\phi(k h), \quad-m \leq k \leq 0,
\end{gathered}
$$

where $h=(1 / m)\left(m \in \mathbb{N}^{+}\right)$stands for stepsize, and $u_{k}$ denotes the approximate value to $u(k h)$. Hence, using Midpoint rule (2.7) to (2.3) yields difference equation

$$
u_{k+1}=u_{k}+h\left[a \tau-b \tau \frac{\left(1 / 2^{n+1}\right)\left(u_{k}+u_{k+1}\right)\left(u_{k-m}+u_{k-m+1}\right)^{n}}{1+\left(1 / 2^{n}\right)\left(u_{k-m}+u_{k-m+1}\right)^{n}}\right] .
$$

Suppose $u_{*}$ is a fixed point of $(2.8)$, then $u_{*}$ satisfies

$$
a\left(1+u_{*}^{n}\right)=b u_{*}^{n+1} .
$$

By the same argument on the unique positive equilibrium point, we know that there exists a unique positive fixed point $u_{*}$. 
Set $y_{k}=u_{k}-u_{*}$. Then $y_{k}$ satisfies

$$
\begin{aligned}
y_{k+1}= & \frac{2^{n} a h \tau+\left(a h \tau-b h \tau u_{*}\right)\left(y_{k-m}+y_{k-m+1}+2 u_{*}\right)^{n}}{2^{n}+(1+b h \tau / 2)\left(y_{k-m}+y_{k-m+1}+2 u_{*}\right)^{n}} \\
& +\frac{2^{n}+(1-b h \tau / 2)\left(y_{k-m}+y_{k-m+1}+2 u_{*}\right)^{n}}{2^{n}+(1+b h \tau / 2)\left(y_{k-m}+y_{k-m+1}+2 u_{*}\right)^{n}} y_{k} .
\end{aligned}
$$

By introducing a new variable $Y_{k}=\left(y_{k}, y_{k-1}, \ldots, y_{k-m}\right)^{T}$, we can rewrite $(2.10)$ as

$$
Y_{k+1}=F\left(Y_{k}, \tau\right) \text {, }
$$

where $F=\left(F_{0}, F_{1}, \ldots, F_{m}\right)^{T}$ and

$$
F_{i}= \begin{cases}\frac{2^{n} a h \tau+\left(a h \tau-b h \tau u_{*}\right)\left(y_{k-m}+y_{k-m+1}+2 u_{*}\right)^{n}}{2^{n}+(1+b h \tau / 2)\left(y_{k-m}+y_{k-m+1}+2 u_{*}\right)^{n}} & \\ +\frac{2^{n}+(1-b h \tau / 2)\left(y_{k-m}+y_{k-m+1}+2 u_{*}\right)^{n}}{2^{n}+(1+b h \tau / 2)\left(y_{k-m}+y_{k-m+1}+2 u_{*}\right)^{n}} y_{k}, & i=0 ; \\ y_{k-i+1}, & 1 \leq i \leq m .\end{cases}
$$

Clearly, the origin is a fixed point of map (2.11), and the linear part of map (2.11) is

$$
Y_{k+1}=A Y_{k}
$$

where

$$
A=\left[\begin{array}{cccccc}
\frac{u_{*}-a h \tau / 2}{u_{*}+a h \tau / 2} & 0 & \cdots & 0 & \frac{-a n h \tau}{2\left(1+u_{*}^{n}\right)\left(u_{*}+a h \tau / 2\right)} & \frac{-a n h \tau}{2\left(1+u_{*}^{n}\right)\left(u_{*}+a h \tau / 2\right)} \\
1 & 0 & \cdots & 0 & 0 & 0 \\
0 & 1 & \cdots & 0 & 0 & 0 \\
\vdots & \vdots & \ddots & \vdots & \vdots & \vdots \\
0 & 0 & \ldots & 1 & 0 & 0 \\
0 & 0 & \ldots & 0 & 1 & 0
\end{array}\right]
$$

whose characteristic equation is

$$
\lambda^{m+1}-\frac{u_{*}-a h \tau / 2}{u_{*}+a h \tau / 2} \lambda^{m}+\frac{n a h \tau}{2\left(1+u_{*}^{n}\right)\left(u_{*}+a h \tau / 2\right)} \lambda+\frac{n a h \tau}{2\left(1+u_{*}^{n}\right)\left(u_{*}+a h \tau / 2\right)}=0 .
$$

Lemma 2.1. All roots of (2.15) have modulus less than one for sufficiently small positive $\tau>0$.

Proof. For $\tau=0,(2.15)$ is equated with

$$
\lambda^{m+1}-\lambda^{m}=0
$$

The equation has an $m$-fold root and a simple root $\lambda=1$. 
Consider the root $\lambda(\tau)$ of (2.15) such that $\lambda(0)=1$. This root depends continuously on $\tau$ and (2.15) is a differential function of $\tau$, from which we have

$$
\begin{aligned}
& \frac{d \lambda}{d \tau}=\frac{-a h(\lambda+1)\left(\lambda^{m}\left(1+u_{*}^{n}\right)+n\right)}{2(m+1) \lambda^{m}\left(1+u_{*}^{n}\right)\left(u_{*}+a h \tau / 2\right)-2 m \lambda^{m-1}\left(1+u_{*}^{n}\right)\left(u_{*}-a h \tau / 2\right)+n a h \tau}, \\
& \frac{d \bar{\lambda}}{d \tau}=\frac{-a h(\bar{\lambda}+1)\left(\bar{\lambda}^{m}\left(1+u_{*}^{n}\right)+n\right)}{2(m+1) \bar{\lambda}^{m}\left(1+u_{*}^{n}\right)\left(u_{*}+a h \tau / 2\right)-2 m \bar{\lambda}^{m-1}\left(1+u_{*}^{n}\right)\left(u_{*}-a h \tau / 2\right)+n a h \tau} .
\end{aligned}
$$

Since $d|\lambda|^{2} / d \tau=\lambda(d \bar{\lambda} / d \tau)+\bar{\lambda}(d \lambda / d \tau)$, so

$$
\left.\frac{d|\lambda|^{2}}{d \tau}\right|_{\tau=0, \lambda=1}=-\frac{2 a h\left(1+n+u_{*}^{n}\right)}{u_{*}\left(1+u_{*}^{n}\right)}<0 .
$$

Consequently, all roots of (2.15) lie in the unit circle for sufficiently small positive $\tau>0$.

A Hopf bifurcation occurs when two roots of the characteristic equation (2.15) cross the unit circle. We have to find values of $\tau$ such that there are roots on the unit circle. The roots on the unit circle are given by $e^{i \omega}, \omega \in(-\pi, \pi]$. Since we are dealing with complex roots of a real polynomial which occur in complex conjugate pairs, we only need to look for $\omega \in(0, \pi]$. For $\omega \in(0, \pi], e^{i \omega}$ is a root of (2.15) if and only if

$$
2\left(1+u_{*}^{n}\right)\left(u_{*}+\frac{a h \tau}{2}\right) e^{i(m+1) \omega}-2\left(1+u_{*}^{n}\right)\left(u_{*}-\frac{a h \tau}{2}\right) e^{i m \omega}+n a h \tau e^{i \omega}+n a h \tau=0 .
$$

So the values of $\tau$ are

$$
\tau=\frac{2 u_{*}\left(1+u_{*}^{n}\right)\left(1-e^{i \omega}\right) e^{i m \omega}}{a h\left(e^{i \omega}+1\right)\left(\left(1+u_{*}^{n}\right) e^{i m \omega}+n\right)}, \quad \omega \in(0, \pi] .
$$

Because $\tau$ is assumed to be real, we get the following relations for $\omega(\in(0, \pi])$ and $\tau$ :

$$
\begin{gathered}
\cos m \omega=-\frac{1+u_{*}^{n}}{n}, \\
\cos \omega=\frac{4\left(1+u_{*}^{n}\right)^{2}\left(u_{*}^{2}+a^{2} h^{2} \tau^{2} / 4\right)-(n a h \tau)^{2}}{4\left(1+u_{*}^{n}\right)^{2}\left(u_{*}^{2}-a^{2} h^{2} \tau^{2} / 4\right)+(n a h \tau)^{2}} .
\end{gathered}
$$

Suppose $\left(1+u_{*}^{n}\right) / n>1$, then $\cos m \omega<-1$, which yields a contradiction. So we have the following result.

Lemma 2.2. Assume that $1+u_{*}^{n}>n$. Then (2.15) has no root with modulus one for all $\tau>0$. 
For $1+u_{*}^{n}<n$, since $\cos m \omega<0$ and $\tau$ is positive real, from (2.20) we know that

$$
\tau=\frac{2 n u_{*}\left(1+u_{*}^{n}\right) \tan (\omega / 2) \sin m \omega}{a h\left(n^{2}-\left(1+u_{*}^{n}\right)^{2}\right)}
$$

Let $\lambda_{i}(\tau)=r_{i}(\tau) e^{i \omega_{i}(\tau)}$ be a root of $(2.15)$ near $\tau=\tau_{i}$ satisfying $r_{i}\left(\tau_{i}\right)=1$ and $\omega_{i}\left(\tau_{i}\right)=\omega_{i}$. Then there are

$$
\begin{gathered}
\omega_{i}=\frac{1}{m}\left[\cos ^{-1}\left(-\frac{1+u_{*}}{n}\right)+2 i \pi\right], \quad i=0,1,2, \ldots,\left[\frac{m-1}{2}\right], \\
\tau_{i}=\tau\left(\omega_{i}\right),
\end{gathered}
$$

where $[\cdot]$ denotes the greatest integer function, and we have the following result.

LEMmA 2.3. If $1+u_{*}^{n}<n$, then

$$
\left.\frac{d r_{i}^{2}(\tau)}{d \tau}\right|_{\tau=\tau_{i}, \omega=\omega_{i}}>0
$$

where $\tau_{i}$ and $\omega_{i}$ satisfy (2.24) and (2.25).

Proof. From (2.15), we notice that

$$
\lambda^{m}=\frac{n a h \tau(\lambda+1)}{\left(1+u_{*}^{n}\right)\left(2 u_{*}(1-\lambda)-a h \tau(1+\lambda)\right)} .
$$

Substituting this equation into (2.17), we have

$$
\frac{d r_{i}^{2}(\tau)}{d \tau}=\lambda \frac{d \bar{\lambda}}{d \tau}+\bar{\lambda} \frac{d \lambda}{d \tau}=\frac{8 m n \tau u_{*}}{\left|a_{1} \lambda^{2}+b_{1} \lambda+c_{1}\right|^{2}}\left[2 u_{*}(n+1)+a h \tau(n-1)-4 u_{*} \cos ^{2} \omega\right]
$$

However,

$$
\begin{aligned}
2 u_{*}(n+1)+a h \tau(n-1)-4 u_{*} \cos ^{2} \omega & \\
= & 2 u_{*}(n+1)+a h \tau(n-1)-4 u_{*}\left[\frac{4\left(1+u_{*}^{n}\right)^{2}\left(u_{*}^{2}+a^{2} h^{2} \tau^{2} / 4\right)-(n a h \tau)^{2}}{4\left(1+u_{*}^{n}\right)^{2}\left(u_{*}^{2}-a^{2} h^{2} \tau^{2} / 4\right)+(n a h \tau)^{2}}\right]^{2} \\
= & \frac{1}{\triangle}\left\{\left(2 u_{*}+a h \tau\right)(n-1)\left[\left(4 u_{*}^{2}\left(1+u_{*}^{n}\right)^{2}\right)^{2}+\left((a h \tau)^{2}\left(n^{2}-\left(1+u_{*}^{n}\right)^{2}\right)\right)^{2}\right]\right. \\
& \left.\quad+4 u_{*}^{2}(a h \tau)^{2}\left(1+u_{*}^{n}\right)^{2}\left[n^{2}-\left(1+u_{*}^{n}\right)^{2}\right]\left[4 u_{*}(n+3)+2 a h \tau(n-1)\right]\right\},
\end{aligned}
$$


where

$$
\triangle=\left|4\left(1+u_{*}^{n}\right)^{2}\left(u_{*}^{2}-\frac{a^{2} h^{2} \tau^{2}}{4}\right)+(n a h \tau)^{2}\right|^{2} .
$$

In view of $1+u_{*}^{n}<n$, we see that

$$
\left.\frac{d r_{i}^{2}(\tau)}{d \tau}\right|_{\tau=\tau_{i}, \omega=\omega_{i}}>0
$$

Thus, the proof is complete.

LEMMA 2.4. (i) If $1+u_{*}^{n}>n$, then all roots of the characteristic equation (2.15) have modulus less than one.

(ii) If $1+u_{*}^{n}<n$, then (2.15) has a pair of simple roots $e^{ \pm i \omega_{i}}$ on the unit circle when $\tau=\tau_{i}, i=0,1,2, \ldots,[(m-1) / 2]$. Furthermore, if $\tau \in\left[0, \tau_{0}\right)$, then all the roots of $(2.15)$ have modulus less than one; if $\tau=\tau_{0}$, then all roots of (2.15) except $e^{ \pm i \omega_{0}}$ have modulus less than one. But if $\tau \in\left(\tau_{i}, \tau_{i+1}\right]$, for $i=0,1,2, \ldots,[(m-1) / 2],(2.15)$ has $2(i+1)$ roots have modulus more than one.

Proof. By Lemmas 2.1 and 2.2, and applying a similar result of Ruan and Wei (see [13, Corollary 2.4]), we arrive at the conclusion (i).

If $1+u_{*}^{n}<n$, let $\tau_{i}$ be as in (2.25). From (2.21) and (2.23), we have that (2.15) has roots $e^{ \pm i \omega_{i}}$ if and only if $\tau=\tau_{i}$ and $\omega=\omega_{i}$ given in (2.24) and (2.25).

Since $\tan (\omega / 2)$ is monotonically increasing for $\omega \in(0, \pi]$ and $(2.24)$, we know that the smallest positive $\tau_{i}$ with roots on unit circle is $\tau_{0}$. By Lemmas 2.1 and 2.3, we know that if $\tau \in\left[0, \tau_{0}\right)$, then all the roots of (2.15) have modulus less than one; if $\tau=\tau_{0}$, then all roots of (2.15) except $e^{ \pm i \omega_{0}}$ have modulus less than one; furthermore, by Rouché's theorem (Dieudonné [14, Theorem 9.17.4]), the statement on the number of eigenvalues with modulus more than one as follows.

Spectral properties in Lemma 2.4 immediately lead to stability properties of the zero solution of (2.10), and equivalently, of the positive fixed point $u=u_{*}$ of (2.8).

THeORem 2.5. (i) If $1+u_{*}^{n}>n$, then $u=u_{*}$ is asymptotically stable for any $\tau \geq 0$.

(ii) If $1+u_{*}^{n}<n$, then $u=u_{*}$ is asymptotically stable for $\tau \in\left[0, \tau_{0}\right)$, and unstable for $\tau>\tau_{0}$.

(iii) For $1+u_{*}^{n}<n$, (2.8) undergoes a Hopf bifurcation at $u_{*}$ when $\tau=\tau_{i}$, for $i=0,1$, $2, \ldots,[(m-1) / 2]$.

\section{Direction and stability of the Hopf bifurcation in discrete model}

In the previous section, we obtained conditions of Hopf bifurcation occurring when $\tau=$ $\tau_{i}, i=0,1,2, \ldots,[(m-1) / 2]$. In this section, we study the direction of the Hopf bifurcation and the stability of the bifurcating periodic solutions when $\tau=\tau_{0}$, using techniques from normal form and center manifold theory (see, e.g., Kuznetsov [12]). To prove the main result, we need some preliminary lemmas. 
Set $\tau=\tau_{0}+\mu, \mu \in R$. Then $\mu=0$ is a Hopf bifurcation value for (2.10). Rewrite (2.10) as

$y_{k+1}$

$$
\begin{aligned}
& =\frac{u_{*}-a h\left(\tau_{0}+\mu\right) / 2}{u_{*}+a h\left(\tau_{0}+\mu\right) / 2} y_{k}-\frac{a n h \tau}{2\left(1+u_{*}^{n}\right)\left(u_{*}+a h\left(\tau_{0}+\mu\right) / 2\right)}\left(y_{k-m}+y_{k-m+1}\right) \\
& +\frac{h a n\left(\tau_{0}+\mu\right)\left[-n+1+(n+1)\left(1+h b\left(\tau_{0}+\mu\right) / 2\right) u_{*}^{n}\right]}{\left(1+u_{*}^{n}\right)^{2}\left(2 u_{*}+h a\left(\tau_{0}+\mu\right)\right)^{2}}\left(y_{k-m}^{2}+y_{k-m+1}^{2}\right) \\
& +\frac{h a n\left(\tau_{0}+\mu\right)\left[-((n+1) /(n+2))\left((n+2) u_{*}^{n}\left(1+h b\left(\tau_{0}+\mu\right) / 2\right)-2(n-1)\right)^{2}+3 n^{2}(n-1) /(n+2)\right]}{\left(1+u_{*}^{n}\right)^{3}\left(2 u_{*}+h a\left(\tau_{0}+\mu\right)\right)^{3}} \\
& \times\left(y_{k-m}^{3}+y_{k-m+1}^{3}\right)+O\left(\left|y^{4}\right|\right) .
\end{aligned}
$$

So system (2.11) is turned into

$$
Y_{k+1}=A Y_{k}+\frac{1}{2} B\left(Y_{k}, Y_{k}\right)+\frac{1}{6} C\left(Y_{k}, Y_{k}, Y_{k}\right)+O\left(\left\|Y_{k}\right\|^{4}\right),
$$

where

$$
\begin{gathered}
B\left(Y_{k}, Y_{k}\right)=\left(b_{0}\left(Y_{k}, Y_{k}\right), 0, \ldots, 0\right), \\
C\left(Y_{k}, Y_{k}, Y_{k}\right)=\left(c_{0}\left(Y_{k}, Y_{k}, Y_{k}\right), 0, \ldots, 0\right), \\
b_{0}(\phi, \psi)=\tilde{b} \cdot\left(\phi_{m-1} \psi_{m-1}+\phi_{m} \psi_{m}\right), \\
c_{0}(\phi, \psi, \eta)=\tilde{c} \cdot\left(\phi_{m-1} \psi_{m-1} \eta_{m-1}+\phi_{m} \psi_{m} \eta_{m}\right),
\end{gathered}
$$

where

$$
\begin{gathered}
\tilde{b}=\frac{n a h\left(\tau_{0}+\mu\right)\left(-(n-1)+(n+1)\left(1+b h\left(\tau_{0}+\mu\right) / 2\right) u_{*}^{n}\right)}{\left(1+u_{*}^{n}\right)^{2}\left(2 u_{*}+a h\left(\tau_{0}+\mu\right)\right)^{2}}, \\
\tilde{c}=\frac{n a h\left(\tau_{0}+\mu\right)\left[-((n+1) /(n+2))\left((n+2) u_{*}^{n}\left(1+b h\left(\tau_{0}+\mu\right) / 2\right)-2(n-1)\right)^{2}+3 n^{2}(n-1) /(n+2)\right]}{\left(1+u_{*}^{n}\right)^{3}\left(2 u_{*}+a h\left(\tau_{0}+\mu\right)\right)^{3}} .
\end{gathered}
$$

Let $q=q\left(\tau_{0}\right) \in C^{m+1}$ be an eigenvector of $A$ corresponding to $e^{i \omega_{0}}$, then

$$
A q=e^{i \omega_{0}} q, \quad A \bar{q}=e^{-i \omega_{0}} \bar{q} .
$$

We also introduce an adjoint eigenvector $q^{*}=q^{*}(\tau) \in C^{m+1}$ having the properties

$$
A^{T} q^{*}=e^{-i \omega_{0}} q^{*}, \quad A^{T} \bar{q}^{*}=e^{i \omega_{0}} \bar{q}^{*},
$$

and satisfying the normalization $\left\langle q^{*}, q\right\rangle=1$, where $\left\langle q^{*}, q\right\rangle=\sum_{i=0}^{m} \bar{q}_{i}^{*} q_{i}$. 
Lemma 3.1 [15]. Define a vector-valued function $q: C \rightarrow C^{m+1}$ by

$$
p(\xi)=\left(\xi^{m}, \xi^{m-1}, \ldots, 1\right)^{T}
$$

If $\xi$ is an eigenvalue of $A$, then $A p(\xi)=\xi p(\xi)$.

In view of Lemma 3.1, we have

$$
q=p\left(e^{i \omega_{0}}\right)=\left(e^{i m \omega_{0}}, e^{i(m-1) \omega_{0}}, \ldots, e^{i \omega_{0}}, 1\right)^{T} .
$$

Lemma 3.2. Suppose $q^{*}=\left(q_{0}^{*}, q_{1}^{*}, \ldots, q_{m}^{*}\right)^{T}$ is the eigenvector of $A^{T}$ corresponding to eigenvalue $e^{-i \omega_{0}}$, and $\left\langle q^{*}, q\right\rangle=1$. Then

$$
q^{*}=\bar{K}\left(\frac{e^{i(m-1) \omega_{0}}}{e^{-i \omega_{0}}-a_{m}}, e^{i(m-1) \omega_{0}}, e^{i(m-2) \omega_{0}}, \ldots, e^{i 2 \omega_{0}}, e^{i \omega_{0}}, \frac{a_{0} e^{i m \omega_{0}}}{e^{-i \omega_{0}}-a_{m}}\right)^{T}
$$

where $a_{m}=\left(2 u_{*}-\tau_{0} a h\right) /\left(2 u_{*}+\tau_{0} a h\right)$, and $a_{0}=a_{1}=-a n h \tau /\left(1+u_{*}^{n}\right)\left(2 u_{*}+a h \tau\right)$ are the coefficients of $\lambda$ in characteristic equation (2.15), and

$$
K=\left[\frac{e^{i \omega_{0}}+a_{0} e^{-i m \omega_{0}}}{e^{i \omega_{0}}-a_{m}}+(m-1)\right]^{-1}
$$

Proof. Assign $q^{*}$ satisfies $A^{T} q^{*}=\bar{z} q^{*}$ with $\bar{z}=e^{-i \omega_{0}}$, then the following identities hold:

$$
\begin{gathered}
a_{m} q_{0}^{*}+q_{1}^{*}=e^{-i \omega_{0}} q_{0}^{*}, \\
q_{k}^{*}=e^{-i \omega_{0}} q_{k-1}^{*}, \quad k=2, \ldots, m-1, \\
a_{1} q_{0}^{*}+q_{m}^{*}=e^{-i \omega_{0}} q_{m-1}^{*}, \\
a_{0} q_{0}^{*}=e^{-i \omega_{0}} q_{m}^{*} .
\end{gathered}
$$

Let $q_{m-1}^{*}=e^{i \omega_{0}} \bar{K}$, then

$$
q^{*}=\bar{K}\left(\frac{e^{i(m-1) \omega}}{e^{-i \omega}-a_{m}}, e^{i(m-1) \omega}, \ldots, e^{i \omega}, \frac{a_{0} e^{i m \omega}}{e^{-i \omega}-a_{m}}\right)^{T} .
$$

From normalization $\left\langle q^{*}, q\right\rangle=1$ and direct computation, the lemma follows.

Let $a(\lambda)$ be characteristic polynomial of $A$ and $\lambda_{0}=e^{i \omega_{0}}$, following the algorithms in [12] and using a computation process similar to that in [15], we can compute an expression for the critical coefficient $c_{1}\left(\tau_{0}\right)$,

$$
c_{1}\left(\tau_{0}\right)=\frac{g_{20} g_{11}\left(1-2 \lambda_{0}\right)}{2\left(\lambda_{0}^{2}-\lambda_{0}\right)}+\frac{\left|g_{11}\right|^{2}}{1-\overline{\lambda_{0}}}+\frac{\left|g_{02}\right|^{2}}{2\left(\lambda_{0}^{2}-\overline{\lambda_{0}}\right)}+\frac{g_{21}}{2},
$$


where

$$
\begin{gathered}
g_{20}=\left\langle q^{*}, B(q, q)\right\rangle, \\
g_{11}=\left\langle q^{*}, B(q, \bar{q})\right\rangle, \\
g_{02}=\left\langle q^{*}, B(\bar{q}, \bar{q})\right\rangle, \\
g_{21}=\left\langle q^{*}, B\left(\bar{q}, \omega_{20}\right)\right\rangle+2\left\langle q^{*}, B\left(q, \omega_{11}\right)\right\rangle+\left\langle q^{*}, C(q, q, \bar{q})\right\rangle, \\
\omega_{20}=\frac{b_{0}(q, q)}{a\left(\lambda_{0}^{2}\right)} p\left(\lambda_{0}^{2}\right)-\frac{\left\langle q^{*}, B(q, q)\right\rangle}{\lambda_{0}^{2}-\lambda_{0}} q-\frac{\left\langle\bar{q}^{*}, B(q, q)\right\rangle}{\lambda_{0}^{2}-\overline{\lambda_{0}}} \bar{q}, \\
\omega_{11}=\frac{b_{0}(q, \bar{q})}{a(1)} p(1)-\frac{\left\langle q^{*}, B(q, \bar{q})\right\rangle}{1-\lambda_{0}} q-\frac{\left\langle\bar{q}^{*}, B(q, \bar{q})\right\rangle}{1-\overline{\lambda_{0}}} \bar{q} .
\end{gathered}
$$

By (3.4), (3.9), and Lemma 3.2, we get

$$
\begin{gathered}
b_{0}\left(\bar{q}, p\left(e^{i 2 \omega_{0}}\right)\right)=\tilde{b}\left(e^{i \omega_{0}}+1\right), \\
b_{0}(q, q)=\tilde{b}\left(e^{i 2 \omega_{0}}+1\right), \\
b_{0}(q, \bar{q})=2 \tilde{b}, \\
c_{0}(q, q, \bar{q})=\tilde{c}\left(e^{i \omega_{0}}+1\right), \\
a\left(e^{i 2 \omega_{0}}\right)=e^{i 2(m+1) \omega_{0}}-a_{m} e^{i 2 m \omega_{0}}-a_{1} e^{i 2 \omega_{0}}-a_{0}, \\
a(1)=1-a_{m}-a_{1}-a_{0}, \\
b_{0}(q, p(1))=\tilde{b}\left(e^{i \omega_{0}}+1\right) .
\end{gathered}
$$

Substituting these into (3.14), we have

$$
\begin{aligned}
c_{1}\left(\tau_{0}\right)= & \frac{m e^{-i m \omega}-(m-1) a_{m} e^{-i(m-1) \omega}+a_{0} e^{-i(2 m-1) \omega}}{2 \Sigma} \\
\times & \times\left[\frac{\tilde{b}^{2}\left(1+e^{i \omega+i 2 \omega}+e^{i 3 \omega}\right)\left(e^{-i 2(m+1) \omega}-a_{m} e^{-i 2 m \omega}-a_{1} e^{-i 2 \omega}-a_{0}\right)}{\delta}\right. \\
& \left.\quad+\frac{4 \tilde{b}^{2}\left(1+e^{i \omega}\right)}{1-a_{m}-a 1-a_{0}}+\tilde{c}\left(1+e^{i \omega}\right)\right]
\end{aligned}
$$

where

$$
\begin{aligned}
& \Sigma=\left|(m-1)\left(e^{i \omega-a_{m}}\right)+e^{i \omega}+e^{-i m \omega} a_{0}\right|^{2}, \\
& \delta=\left|e^{i 2(m+1) \omega}-a_{m} e^{i 2 m \omega}-a_{1} e^{i 2 \omega}-a_{0}\right|^{2} .
\end{aligned}
$$

Lemma 3.3 [15]. Given the map (2.11) and assume

(1) $\lambda(\tau)=r(\tau) e^{i \omega(\tau)}$, where $r\left(\tau^{*}\right)=1, r^{\prime}\left(\tau^{*}\right) \neq 0$, and $\omega\left(\tau^{*}\right)=\omega^{*}$;

(2) $e^{i k \omega^{*}} \neq 1$ for $k=1,2,3,4$;

(3) $\operatorname{Re}\left[e^{-i \omega^{*}} c_{1}\left(\tau^{*}\right)\right] \neq 0$, 
Table 4.1. The values of $\tau_{k}$.

\begin{tabular}{c|ccccccc}
\hline & $\tau_{0}$ & $\tau_{1}$ & $\tau_{2}$ & $\tau_{3}$ & $\tau_{4}$ & $\tau_{5}$ & $\cdots$ \\
\hline$h=\frac{1}{2}$ & 4.63837 & - & - & - & - & - & - \\
$h=\frac{1}{10}$ & 4.13355 & 16.3729 & 32.8878 & 63.4695 & 391.224 & - & - \\
$h=\frac{1}{100}$ & 4.11548 & 15.3644 & 26.6438 & 37.9761 & 110.292 & 136.042 & $\ldots$ \\
\hline
\end{tabular}

Table 4.2. The values of $c_{1}\left(\tau_{0}\right)$ and $\operatorname{Re}\left[e^{-i \omega_{0}} c_{1}\left(\tau_{0}\right)\right]$.

\begin{tabular}{l|cc}
\hline & $c_{1}\left(\tau_{0}\right)$ & $\operatorname{Re}\left[e^{-i \omega_{0}} c_{1}\left(\tau_{0}\right)\right]$ \\
\hline$h=\frac{1}{2}$ & $0.0107231-0.128588 i$ & -0.113006 \\
$h=\frac{1}{10}$ & $-0.0344386-0.0198785 i$ & -0.0380641 \\
$h=\frac{1}{100}$ & $-0.00396203-0.0010418 i$ & -0.00398495 \\
\hline
\end{tabular}

then an invariant closed curve, topologically equivalent to a circle, for map (2.11) exists for $\tau$ in a one side neighborhood of $\tau^{*}$. The radius of the invariant curve grows like $O\left(\sqrt{\left|\tau-\tau^{*}\right|}\right)$. One of the four cases below applies:

(1) $r^{\prime}\left(\tau^{*}\right)>0, \operatorname{Re}\left[e^{-i \omega^{*}} c_{1}\left(\tau^{*}\right)\right]<0$. The origin is asymptotically stable for $\tau<\tau^{*}$ and unstable for $\tau>\tau^{*}$. An attracting invariant closed curve exists for $\tau>\tau^{*}$.

(2) $r^{\prime}\left(\tau^{*}\right)>0, \operatorname{Re}\left[e^{-i \omega^{*}} c_{1}\left(\tau^{*}\right)\right]>0$. The origin is asymptotically stable for $\tau<\tau^{*}$ and unstable for $\tau>\tau^{*}$. A repelling invariant closed curve exists for $\tau<\tau^{*}$.

(3) $r^{\prime}\left(\tau^{*}\right)<0, \operatorname{Re}\left[e^{-i \omega^{*}} c_{1}\left(\tau^{*}\right)\right]<0$. The origin is asymptotically stable for $\tau>\tau^{*}$ and unstable for $\tau<\tau^{*}$. An attracting invariant closed curve exists for $\tau<\tau^{*}$.

(4) $r^{\prime}\left(\tau^{*}\right)<0, \operatorname{Re}\left[e^{-i \omega^{*}} c_{1}\left(\tau^{*}\right)\right]>0$. The origin is asymptotically stable for $\tau>\tau^{*}$ and unstable for $\tau<\tau^{*}$. An attracting invariant closed curve exists for $\tau>\tau^{*}$.

From the discussion in Section 2, we know that $r^{\prime}\left(\tau^{*}\right)>0$, therefore, by Lemma 3.3 we have the following result.

Theorem 3.4. If $1+u_{*}^{n}<n$, then $u=u_{*}$ is asymptotically stable for $\tau \in\left[0, \tau_{0}\right)$, and unstable for $\tau>\tau_{0}$. An attracting (repelling) invariant closed curve exists for $\tau>\tau_{0}$ if $\operatorname{Re}\left[e^{-i \omega_{0}} c_{1}\left(\tau_{0}\right)\right]$ $<0(>0)$.

\section{Numerical test}

Firstly, we choose system (2.1) with $b=1, a=16 / 9, n=3$, and initial value $u=2+\sin (t)$, then $u_{*}=2$ and satisfy $1+u_{*}^{n}>n$. According to Lemma 2.1, we deduce that for this case $u_{*}=2$ is asymptotically stable for any $\tau \geq 0$ (Figure 4.1 ). Secondly, we consider system (2.1) with $b=1, a=0.5, n=3$, and initial value $u=2+\sin (t)$, then $u_{*}=1$ and satisfy 
12 Discrete Dynamics in Nature and Society

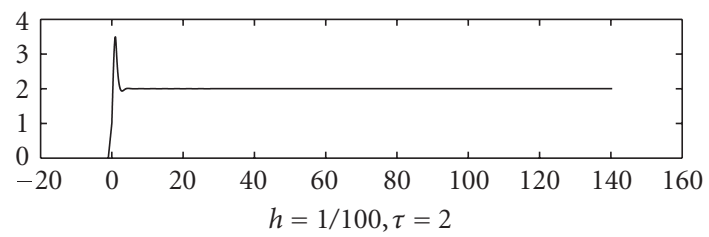

(a)

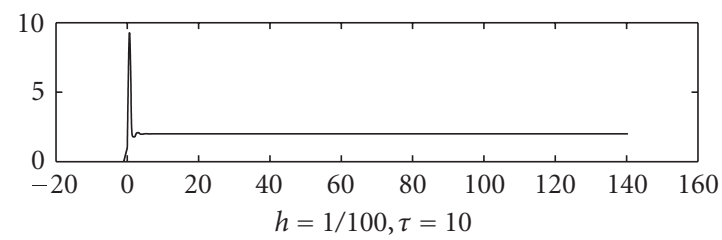

(b)

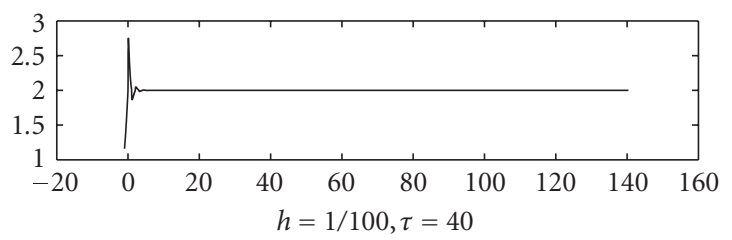

(c)

Figure 4.1. $b=1, a=16 / 9, n=3, h=1 / 100$.

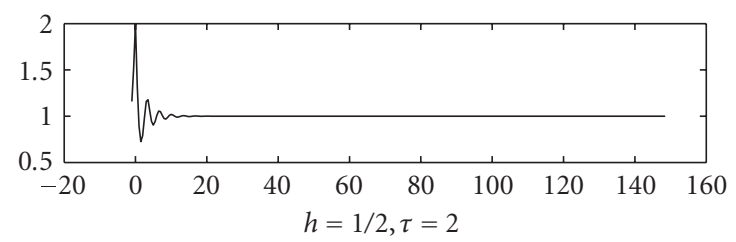

(a)

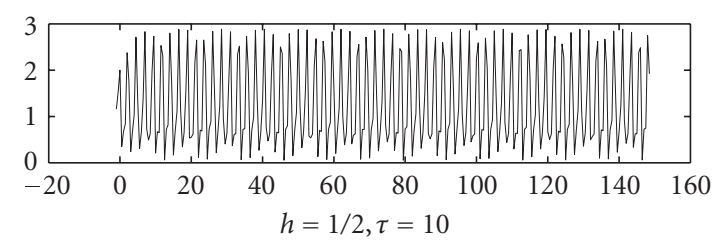

(b)

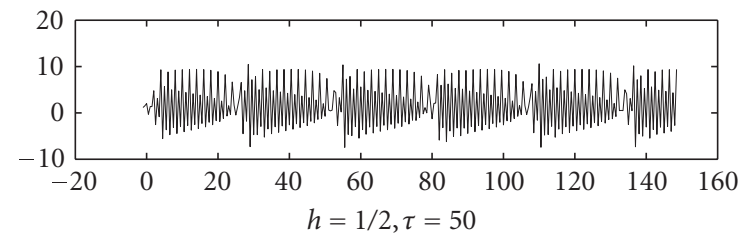

(c)

Figure 4.2. $\tau_{0}=4.63837, \operatorname{Re}\left[e^{-i \omega_{0}} c_{1}\left(\tau_{0}\right)\right]=-0.113006<0$. 


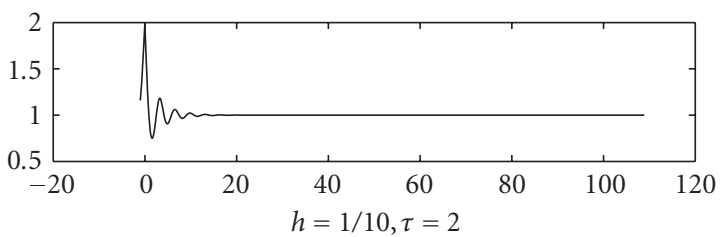

(a)

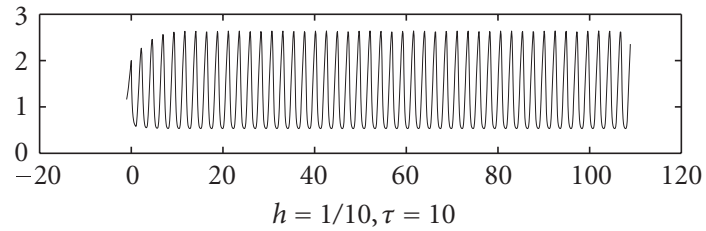

(b)

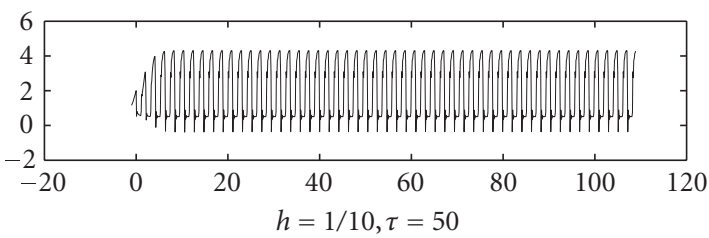

(c)

Figure 4.3. $\tau_{0}=4.13355, \operatorname{Re}\left[e^{-i \omega_{0}} c_{1}\left(\tau_{0}\right)\right]=-0.0380641<0$.

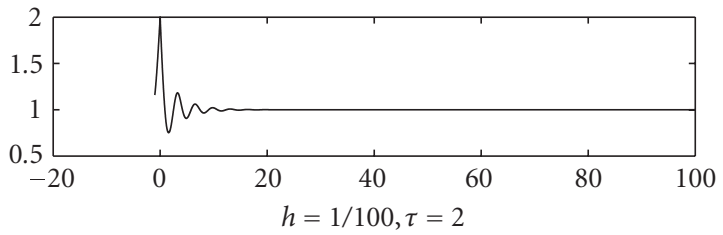

(a)

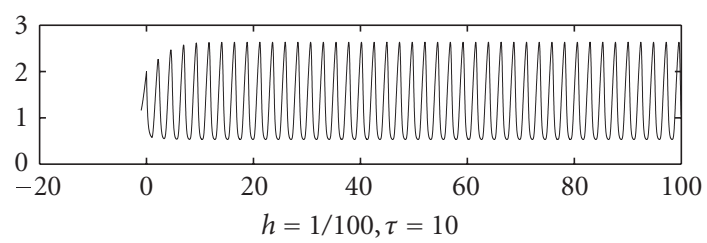

(b)

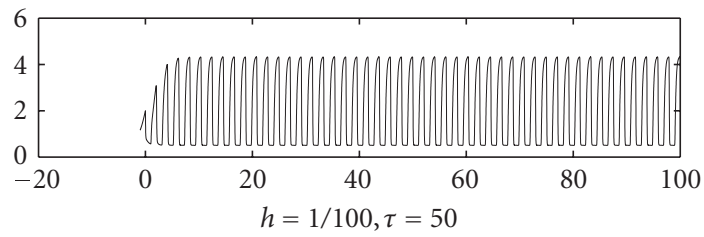

(c)

Figure 4.4. $\tau_{0}=4.11548, \operatorname{Re}\left[e^{-i \omega_{0}} \mathcal{c}_{1}\left(\tau_{0}\right)\right]=-0.00398495<0$. 


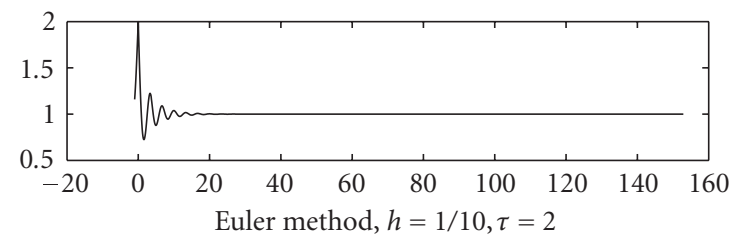

(a)

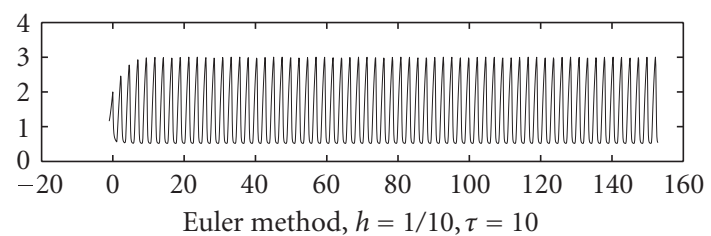

(b)

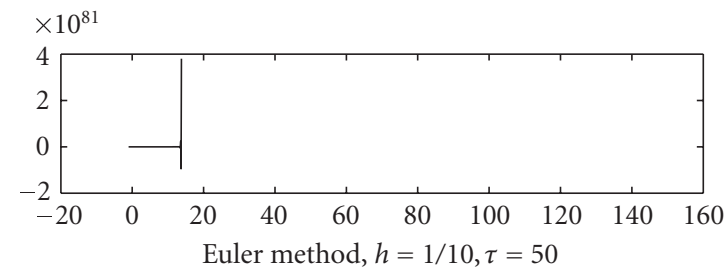

(c)

Figure 4.5. The solution of system (1.1) applied by Euler method with $b=1, a=0.5, n=3$, and $h=1 / 10$ is invalid for $\tau=50$.

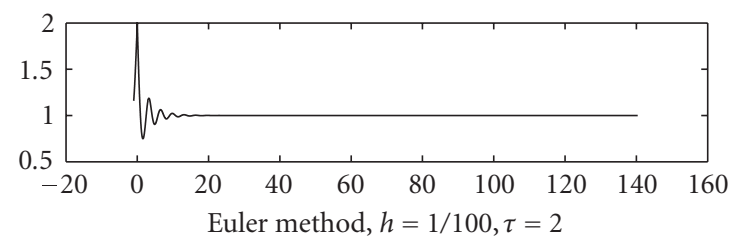

(a)

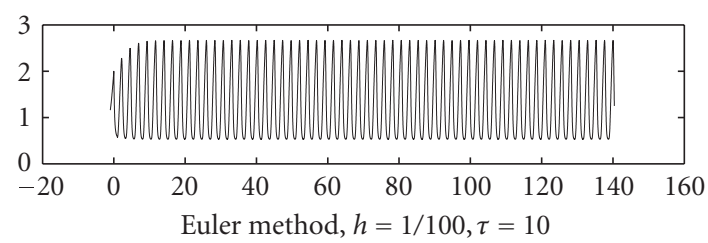

(b)

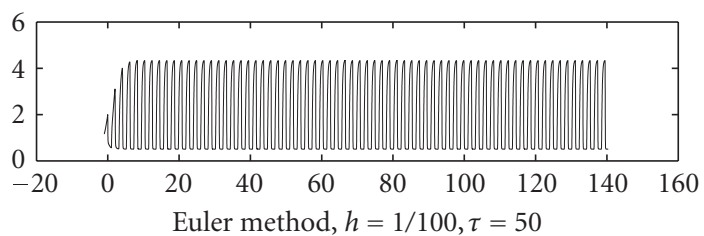

(c)

Figure 4.6. The solution of system (1.1) applied by Euler method with $b=1, a=0.5, n=3$, and $h=1 / 100$. 
$1+u_{*}^{n}<n$. From Table 4.1, we see that there exists a sequence of $\tau_{i}$, and with the increasing of $m \tau_{0}$ asymptotically convergence to $\tau_{0} \approx 4$.1153, which is the true value. Furthermore, noting Lemma 2.1 we judge that $u_{*}=1$ is asymptotically stable for $\tau \in\left[0, \tau_{0}\right)$ and unstable for $\tau>\tau_{0}$, and system (2.1) undergoes a Hopf bifurcation at $u_{*}$ when $\tau=\tau_{i}$, for $i=0,1,2, \ldots,[(m-1) / 2]$. Combining Table 4.1 with Table 4.2 , and by Lemma 3.1, we can derive that an attracting invariant closed curve exists for $\tau>\tau_{0}$ for $\operatorname{Re}\left[e^{-i \omega_{0}} c_{1}\left(\tau_{0}\right)\right]<0$. Justly, Figures $4.2,4.3$, and 4.4 coincide with those results.

In this section, we take two systems to verify Theorems 2.5 and 3.4. What we get shows that our theoretical analysis is correct.

At last, in order to illustrate the merits of Midpoint Rule we try to use another methods to get discrete systems of (1.1), such as explicit Euler method. Compare Figures 4.3 and 4.5 , we can find that for $\tau=50$, using the same step size $h=1 / 10$, the Euler discrete model cannot get the bifurcation solution, if we will show the realities, we must reduce the step size (see Figure 4.6). But the Midpoint discrete can get it. These show that, $\tau_{0}$ in discrete system (2.8) converges the Hopf bifurcation of the original system more fast than ones in another discrete system, with the same step size.

\section{Conclusions}

From the above analysis, we can find that the delay does not influence the system's stability when the coefficients of system satisfy condition $1+u_{*}^{n}>n$. But when the coefficients of system satisfy condition $1+u_{*}^{n}<n$, for small delay the positive fixed point of system is stable. With the increasing of delay, the positive fixed point loses its stability and a family of periodic solutions occurs. We could compute the bifurcation for a given system (1.1). Therefore, we infer that when the time between oxygenation of blood in the lungs and stimulation of chemoreceptors in the brainstem is less than $\tau_{0}$, the arterial $\mathrm{CO}_{2}$ concentration in the patient will attain stability; but when the time is more than $\tau_{0}$, the arterial $\mathrm{CO}_{2}$ concentration in the person will be unstable, that is, the patient has an abnormal respiration (Cheyne-Stokes respiration).

\section{Acknowledgment}

This paper is supported by the National Natural Science Foundation of China (10271036) and of HIT(200518).

\section{References}

[1] M. C. Mackey and L. Glass, "Oscillation and chaos in physiological control systems," Science, vol. 197, no. 4300, pp. 287-289, 1977.

[2] M. C. Khoo, R. E. Kronauer, K. P. Strohl, and A. S. Slutsky, "Factors inducing periodic breathing in humans: a general model," Journal of Applied Physiology, vol. 53, no. 3, pp. 644-659, 1982.

[3] H. T. Milhorn Jr., R. Benton, R. Ross, and A. C. Guyton, "A mathematical model of the human respiratory control system," Biophysical Journal, vol. 5, no. 1, pp. 27-46, 1965.

[4] G. S. Longobardo, N. S. Cherniack, and A. P. Fishman, "Cheyne-Stokes breathing producted by a model the human respiratory system," Journal of Applied Physiology, vol. 21, no. 6, pp. 18391846, 1966. 
[5] N. S. Cherniack and G. S. Longobardo, "Cheyne-Stokes breathing. An instability in physiologic control," The New England Journal of Medicine, vol. 288, no. 18, pp. 952-957, 1973.

[6] V. Wulf and N. J. Ford, "Numerical Hopf bifurcation for a class of delay differential equations," Journal of Computational and Applied Mathematics, vol. 115, no. 1-2, pp. 601-616, 2000.

[7] A. Bellen and M. Zennaro, Numerical Methods for Delay Differential Equations, Numerical Mathematics and Scientific Computation, The Clarendon Press, Oxford University Press, New York, NY, USA, 2003.

[8] K. Feng, Collected Works of Feng Kang (II), National Defence Industry Press, Beijing, China, 1995.

[9] E. Hairer and G. Wanner, Solving Ordinary Differential Equations II: Stiff and Differential Algebraic Equations, Springer, New York, NY, USA, 1993.

[10] B. D. Hassard, N. D. Kazarinoff, and Y. H. Wan, Theory and Applications of Hopf Bifurcation, vol. 41 of London Mathematical Society Lecture Note Series, Cambridge University Press, Cambridge, UK, 1981.

[11] V. Wulf and N. J. Ford, "Numerical Hopf bifurcation for a class of delay differential equations," Journal of Computational and Applied Mathematics, vol. 115, no. 1-2, pp. 601-616, 2000.

[12] Y. A. Kuznetsov, Elements of Applied Bifurcation Theory, vol. 112 of Applied Mathematical Sciences, Springer, New York, NY, USA, 1995.

[13] S. Ruan and J. Wei, "On the zeros of transcendental functions with applications to stability of delay differential equations with two delays," Dynamics of Continuous, Discrete \& Impulsive Systems. Series A. Mathematical Analysis, vol. 10, no. 6, pp. 863-874, 2003.

[14] J. Dieudonné, Foundations of Modern Analysis, vol. 10 of Pure and Applied Mathematics, Academic Press, New York, NY, USA, 1960.

[15] V. Wulf, Numerical analysis of delay differential equations undergoing a Hopf bifurcation, Ph.D. thesis, University of Liverpool, Liverpool, UK, 1999.

Xiaohua Ding: Department of Mathematics, Harbin Institute of Technology, Weihai 264209, China Email addresses: mathdxh@126.com; mathdxh@hit.edu.cn

Huan Su: Department of Mathematics, Harbin Institute of Technology, Weihai 264209, China

Email address: suhuan1981@yahoo.com.cn 


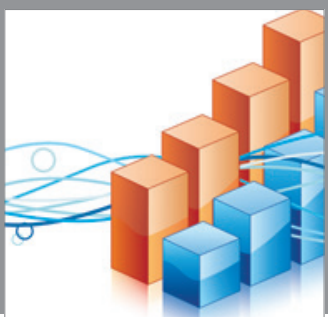

Advances in

Operations Research

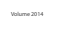

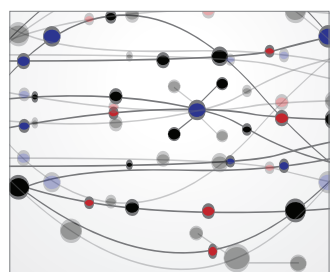

\section{The Scientific} World Journal
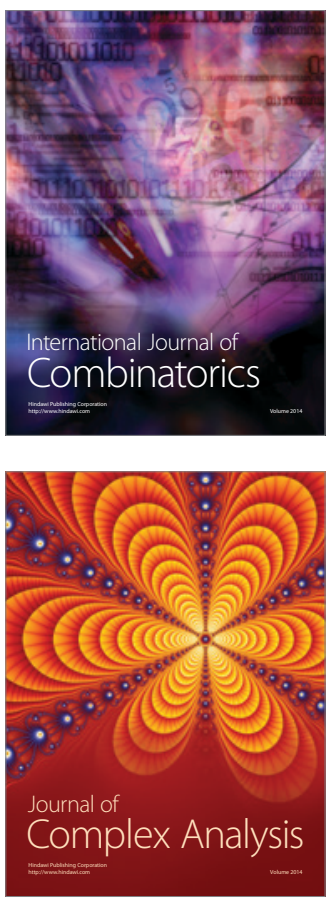

International Journal of

Mathematics and

Mathematical

Sciences
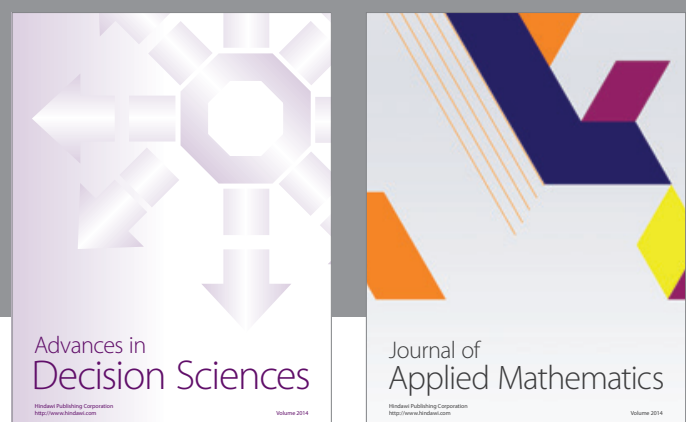

Journal of

Applied Mathematics
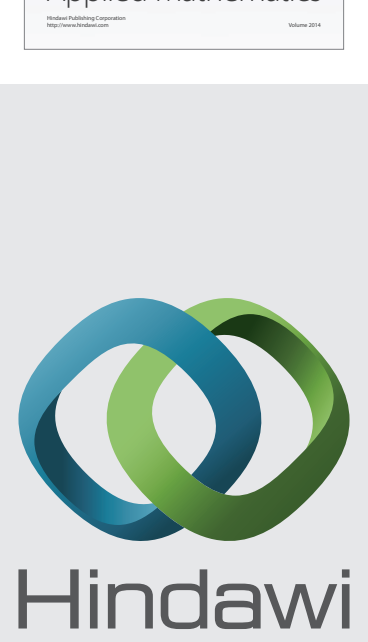

Submit your manuscripts at http://www.hindawi.com
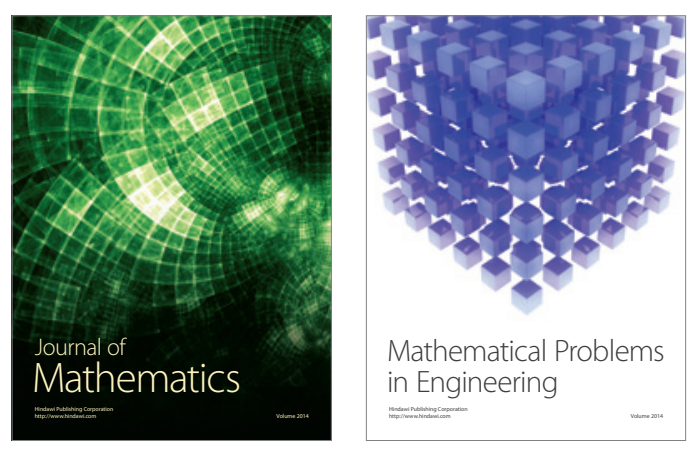

Mathematical Problems in Engineering
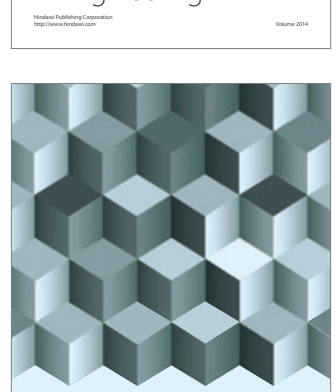

Journal of

Function Spaces
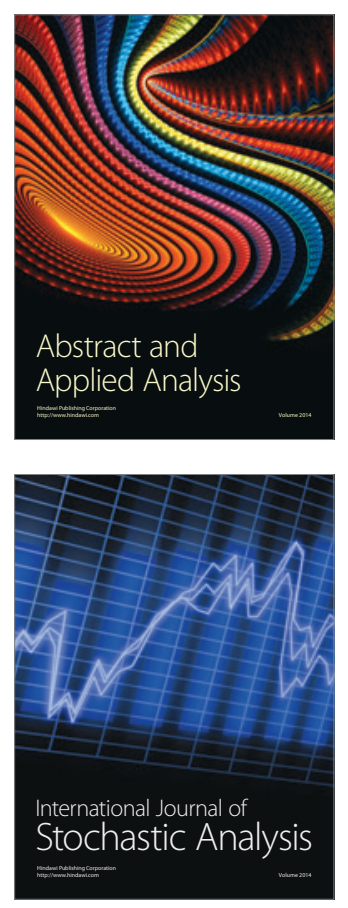

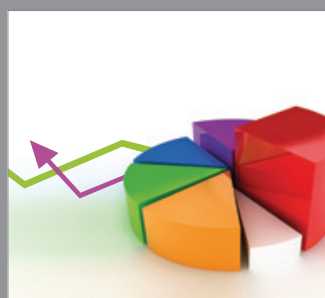

ournal of

Probability and Statistics

Promensencen
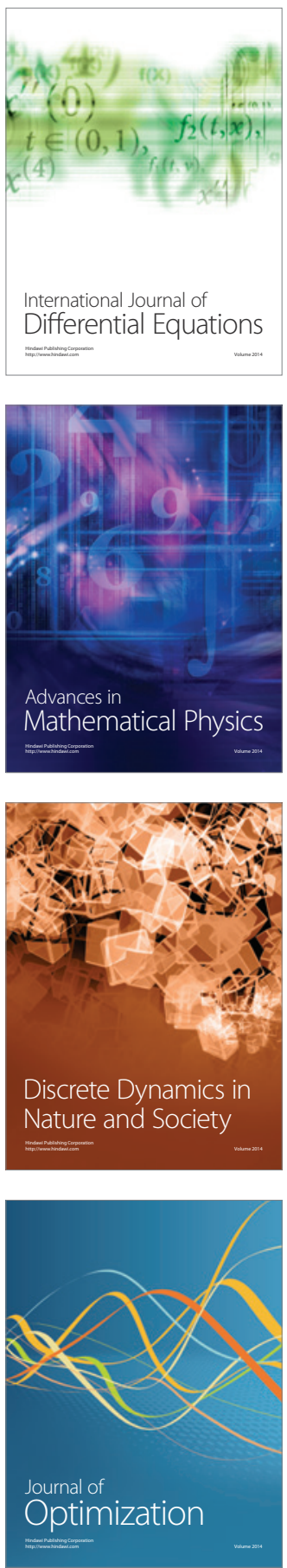